University of Wollongong

Research Online

Faculty of Engineering and Information

Faculty of Engineering and Information

Sciences - Papers: Part A

Sciences

$1-1-2013$

A time-varying MIMO generalized minimum variance controller for servo application

\author{
Zheng Li \\ University of Wollongong, zli@uow.edu.au \\ Guoli Wang \\ Sun Yat-Sen University
}

Follow this and additional works at: https://ro.uow.edu.au/eispapers

Part of the Engineering Commons, and the Science and Technology Studies Commons

Research Online is the open access institutional repository for the University of Wollongong. For further information contact the UOW Library: research-pubs@uow.edu.au 


\title{
A time-varying MIMO generalized minimum variance controller for servo application
}

\author{
Abstract \\ A generalized minimum variance controller is developed for multiple input and multiple output systems \\ having time-varying dynamics. The plant to be controlled is described using a controlled autoregressive \\ moving average model and the control objective is to minimize a generalized minimum variance \\ performance index for servo applications. (2013) Trans Tech Publications, Switzerland.

\section{Keywords} \\ application, generalized, controller, mimo, varying, variance, minimum, time, servo \\ Disciplines \\ Engineering | Science and Technology Studies

\section{Publication Details} \\ Z. Li \& G. Wang, "A time-varying MIMO generalized minimum variance controller for servo application," \\ Applied Mechanics and Materials, vol. 321-324, (2013) pp. 1593-1596, 2013.
}




\title{
A Time-Varying MIMO Generalized Minimum Variance Controller for Servo Application
}

\author{
Zheng $\mathrm{Li}^{1, \mathrm{a}}$ and Guoli Wang ${ }^{2, \mathrm{~b}}$ \\ ${ }^{1}$ School of Electrical, Computer and Telecommunication Engineering, University of Wollongong, \\ Wollongong NSW 2522, AUSTRALIA \\ ${ }^{2}$ Department of Automation, Sun Yat-Sen University, Guangzhou, China \\ azli@uow.edu.au, isswgl@mail.sysu.edu.cn
}

Keywords: Adaptive control; multiple variable systems, generalized minimum variance control; time-varying systems.

\begin{abstract}
A generalized minimum variance controller is developed for multiple input and multiple output systems having time-varying dynamics. The plant to be controlled is described using a controlled autoregressive moving average model and the control objective is to minimize a generalized minimum variance performance index for servo applications.
\end{abstract}

\section{Introduction}

There are growing interests in applying generalized minimum variance controllers (GMVCs) for DC motor control for servo applications [1-3]. A linear time-varying (LTV) GMVC was recently developed for LTV single input and single output (SISO) systems for servo applications without using the pseudocommutation [4]. In this paper we extend this LTV GMVC for multiple input and multiple output (MIMO) LTV plants for GMVC. The LTV plants are described using a MIMO LTV transfer operator that is a natural extension of the transfer functions of linear time-invariant (LTI) plants. Incremental inputs are used in the cost functional for dealing with large deterministic disturbances that are common in industrial applications. The stochastic nature of the processes is taken into account in the form of variance of the output tracking error.

\section{Control Objective}

The plants to be controlled have $p$ inputs and $p$ outputs. It is described using the following LTV MIMO controlled autoregressive moving average (CARMA) model.

$$
A\left(k, q^{-1}\right) Y(k+d)=B\left(k, q^{-1}\right) U(k)+C\left(k, q^{-1}\right) W(k+d),
$$

where $U(k)$ and $Y(k)$ are $p \times 1$ plant input and output, $d$ is the time delay between them, $W(k)$ is a $p \times 1$ zero mean, independent Gaussian process. The variance of $W(k)$ is assumed to be a $p \times p$ time-varying matrix that is uniformly bounded away from infinite. In the CARMA model $q^{-1}$ is the one-step-delay operator such that $q^{-1} f(k)=f(k-1)$ and

$$
\begin{aligned}
& A\left(k, q^{-1}\right)=I+A_{1}(k) q^{-1}+A_{2}(k) q^{-2}+\ldots+A_{n}(k) q^{-n} \\
& B\left(k, q^{-1}\right)=B_{0}(k)+B_{1}(k) q^{-1}+B_{2}(k) q^{-2}+\ldots+B_{m}(k) q^{-m} \\
& C\left(k, q^{-1}\right)=I+C_{1}(k) q^{-1}+\ldots+C_{h}(k) q^{-h}
\end{aligned}
$$

are $p \times p$ LTV moving average operators (MAO's) where $A_{i}(k), B_{j}(k)$ and $C_{r}(k), i=1,2, \ldots, n, j=0$, $1, \ldots, m, r=1,2, \ldots, h$, are $p \times p$ time-varying matrices uniformly bounded away from infinite. It is also assumed that the determinant of $B_{0}(k)$ is uniformly bounded away from zero. 
The inverse operation of an LTV MAO is defined as an LTV autoregressive operator (ARO) with the notation $A^{-1}\left(k, q^{-1}\right)$ [5]. When an LTV ARO is exponentially stable the following cancellation is valid as in the LTI case.

$$
\begin{aligned}
& A\left(k, q^{-1}\right) A^{-1}\left(k, q^{-1}\right) V(k)=V(k) \\
& A^{-1}\left(k, q^{-1}\right) A\left(k, q^{-1}\right) V(k)=V(k)+\varepsilon(k),
\end{aligned}
$$

where $\varepsilon(k)$ satisfies $A\left(k, q^{-1}\right) \varepsilon(k)=0 . \varepsilon(k)$ is a zero input solution to the autoregressive equation and decays exponentially to zero because of the exponential stability. It will be suppressed in the rest part of this paper for simplicity of notation. For the design of the LTV GMVC we assume that both $A^{-1}\left(k, q^{-1}\right)$ and $C^{-1}\left(k, q^{-1}\right)$ are exponentially stable and all the plant parameters in the MIMO LTV CARMA model are known.

Given a $p \times 1$ uniformly bounded reference $Z(k)$ the generalised minimum variance control objective is to minimise the following GMVC performance index by generating a sequence of control, $U(k)$.

$$
\begin{aligned}
& J(k+d)=\boldsymbol{E}\left\{[\Psi(k+d)-S(k+d)]^{T} Q(k)[\Psi(k+d)-S(k+d)]\right. \\
& \left.+[U(k)-U(k-1)]^{T} R(k)[U(k)-U(k-1)] \mid D(k)\right\}
\end{aligned}
$$

where

$$
\Psi(k+d)=P^{-1}\left(k, q^{-1}\right) y(k+d), \quad S(k+d)=Q^{-1}\left(k, q^{-1}\right) Z(k+d) .
$$

$P^{-1}\left(k, q^{-1}\right)$ and $Q^{-1}\left(k, q^{-1}\right)$ are exponentially stable LTV filters of digrees $n_{p}$ and $n_{q}$ and $P\left(k, q^{-1}\right)$ is a monic LTV MAO. $E$ is the operator for mathematical expectation, the superscript, $T$, denotes matrix transpose and $D(k)$ is the set of input and output data up to and including the current time $k$. $P(k)$ and $R(k)$ are uniformly positive definite matrices that are uniformly bounded away from infinite. They are weighting matrices for the output tracking error and incremental in plant input.

\section{GMVC}

Left dividing $C\left(k, q^{-1}\right)$ using $A\left(k, q^{-1}\right) P\left(k, q^{-1}\right)$ we have the following matrix equation.

$$
C\left(k, q^{-1}\right)=A\left(k, q^{-1}\right) P\left(k, q^{-1}\right) F\left(k, q^{-1}\right)+G\left(k, q^{-1}\right) q^{-d}
$$

where

$$
F\left(k, q^{-1}\right)=I+F_{1}(k) q^{-1}+\ldots+F_{d-1}(k) q^{-d+1}
$$

is the quotient,

$$
G\left(k, q^{-1}\right)=G_{0}(k)+G_{1}(k) q^{-1}+\ldots+G_{s}(k) q^{-s}
$$

is the remainder and $s=\max \left(h-d, n+n_{p}+1\right)$. The quotient and remainder can be determined uniquely from equation (6) using long division. Substitute (6) into (1) we have

$$
A\left(k, q^{-1}\right) Y(k+d)=B\left(k, q^{-1}\right) U(k)+A\left(k, q^{-1}\right) P\left(k, q^{-1}\right) F\left(k, q^{-1}\right) W(k+d)+G\left(k, q^{-1}\right) W(k) .
$$

Left dividing by $A\left(k, q^{-1}\right) P\left(k, q^{-1}\right)$ and taking mathematical expectation on both sides of the above equation conditioned on $D(k)$ we have the following $d$-step-ahead minimum variance prediction of the plant output. 


$$
\hat{\Psi}(k+d)=\left[A\left(k, q^{-1}\right) P\left(k, q^{-1}\right)\right]^{-1}\left[B\left(k, q^{-1}\right) U(k)+G\left(k, q^{-1}\right) W(k)\right] .
$$

where $\hat{\Psi}(k+d)=E[\Psi(k+d) / D(k)]$.

GMVC Theorem. If the LTV AROs $A^{-1}\left(k, q^{-1}\right)$ and $C^{-1}\left(k, q^{-1}\right)$ are exponentially stable, the LTV GMVC for the CARMA model is given by

$$
\begin{aligned}
\hat{W}(k)= & C^{-1}\left(k-d, q^{-1}\right)\left[A\left(k-d, q^{-1}\right) Y(k)-B\left(k-d, q^{-1}\right) U(k-d)\right], \\
U(k)= & T^{-1}\left(k, q^{-1}\right)\left[A\left(k, q^{-1}\right) P\left(k, q^{-1}\right) Q^{-1}\left(k, q^{-1}\right) Z(k+d)-G\left(k, q^{-1}\right) \hat{W}(k)\right] \\
& \left.+A\left(k, q^{-1}\right) P\left(k, q^{-1}\right) Q^{-1}(k) B_{0}^{-T}(k) R(k) U(k-1)\right]
\end{aligned}
$$

where

$$
T\left(k, q^{-1}\right)=B\left(k, q^{-1}\right)+A\left(k, q^{-1}\right) P\left(k, q^{-1}\right) Q^{-1}(k) B_{0}^{-T}(k) R(k) .
$$

Proof. Compare (9) and (10) we have

$$
\hat{\Psi}(k+d)=\Psi(k+d)-F\left(k, q^{-1}\right) W(k+d) .
$$

Substituting the above prediction into the GMVC index we have

$$
\begin{aligned}
J(k+d)= & {[\hat{\Psi}(k+d)-S(k+d)]^{T} P(k)[\hat{\Psi}(k+d)-S(k+d)]+\left[U^{T}(k)-U^{T}(k-1)\right] R(k)[U(k)-U(k-1)] } \\
& +E\left\{\left[F\left(k, q^{-1}\right) W(k+d)\right]^{T} P(k)\left[F\left(k, q^{-1}\right) W(k+d)\right] \mid D(k)\right\} .
\end{aligned}
$$

It follows form (1) that

$$
\frac{\partial J(k+d)}{\partial U(k)}=2 B_{0}^{T}(k) Q(k)[\hat{\Psi}(k+d)-S(k+d)]+2 R(k)[U(k)-U(k-1)]
$$

and

$$
\frac{\partial^{2} J(k+d)}{\partial U^{2}(k)}=2 B_{0}^{T}(k) Q(k) B_{0}(k)+2 R(k) .
$$

Therefore, there exists optimal control $U(k)$ such that the generalized minimum variance cost functional will be achieved because the second order derivative is uniformly positive definite. Letting (14) zero the optimal control can be determined as the following.

$$
Q^{-1}(k) B_{0}^{-T}(k) R(k)[U(k)-U(k-1)]=S(k+d)-\hat{\Psi}(k+d) .
$$

It follows from (10) that

$$
\begin{aligned}
& Q^{-1}(k) B_{0}^{-T}(k) R(k)[U(k)-U(k-1)]=S(k+d)-\left[A\left(k, q^{-1}\right) P\left(k, q^{-1}\right)\right]^{-1}\left[B\left(k, q^{-1}\right) U(k)\right. \\
& \left.\quad+G\left(k, q^{-1}\right) W(k)\right]
\end{aligned}
$$

where $W(k)$ can be estimated by inverting (1) to have (11a). Solving for $U(k)$ and replacing $W(k)$ using its estimate we have the GMVC controller (11b and 11c). Comparing (1) with (11a) we have 


$$
C\left(k, q^{-1}\right) \tilde{W}(k+d)=0
$$

where

$$
\tilde{W}(k+d)=W(k+d)-\hat{W}(k+d)
$$

is the estimation error of (11a). Because of the exponential stability of $C^{-1}\left(k, q^{-1}\right)$ this error will always decay exponentially to zero regardless initial conditions. As a result, $U(k)$ will also converge exponentially to the optimal control. Noting (1,11b, 18 and 19) we have the closed loop equation for the LTV GMVC

$$
\begin{gathered}
{\left[\begin{array}{ccc}
C\left(k-d, q^{-1}\right) & 0 & 0 \\
-G\left(k, q^{-1}\right) & H\left(k, q^{-1}\right) & 0 \\
0 & -B\left(k-d, q^{-1}\right) q^{-d} & A\left(k-d, q^{-1}\right)
\end{array}\right]\left[\begin{array}{c}
\tilde{W}(k) \\
U(k) \\
Y(k)
\end{array}\right]} \\
\quad=\left[\begin{array}{cc}
0 & 0 \\
-G\left(k, q^{-1}\right) & A\left(k, q^{-1}\right) P\left(k, q^{-1}\right) Q^{-1}\left(k, q^{-1}\right) \\
C\left(k-d, q^{-1}\right) & 0
\end{array}\right]\left[\begin{array}{c}
W(k) \\
Z(k+d)
\end{array}\right]
\end{gathered}
$$

where

$$
H\left(k, q^{-1}\right)=B\left(k, q^{-1}\right)+A\left(k, q^{-1}\right) P\left(k, q^{-1}\right) Q^{-1}(k) B_{0}^{-T}(k) R(k)\left(1-q^{-1}\right) .
$$

The inverse of the square matrix on the left of the closed-loop equation (20) determines the closed-loop stability because it is the AR operator of the closed-loop equation. Because it is diagonal and both $A^{-1}\left(k, q^{-1}\right)$ and $C^{-1}\left(k, q^{-1}\right)$ are exponentially stable the closed-loop system is exponentially stable if $H^{-1}\left(k, q^{-1}\right)$ is exponentially stable.

\section{Conclusions}

An MIMO LTV GMVC is developed for MIMO LTV systems for servo applications without using pseudocommutation. Two weighting matrices are used in order to reduce the output tracking error and fluctuation in the plant input at the same time. The input incremental is also introduced here to allow large control action for more accurate output tracking. It reduces also the speed of inputs for less fluctuation.

\section{References}

[1] S. Donoghue, J. W. Finch, D. Giaouris and A. Jones: Generalized Minimum Variance Controller as a Velocity Loop Controller of a Casting Drum Drive in a Polyester Manufacturing Line, Proceedings of the World Congress on Engineering 2008, Vol III, WCE (2008).

[2] M. Ezzaraa, M. A. Lafkih and M. Ramzi: Dj.M. Maric, P.F. Meier and S.K. Estreicher: Adaptive Generalized Minimum Variance of DC Motor, European Journal of Scientific Research, Vol.67 No.1 pp. 66-74 (2011).

[3] S. C. Mahato and J. P. Tiwari: Adaptive Generalized Minimum Variance Control of DC Motor, International Journal of Engineering Research \& Technology (IJERT), Vol. 1 Issue 5 (2012)

[4] Z. Li: A Time-Varying Generalized Minimum Variance Controller for Servo Application, Applied Mechanics and Materials, Vol. 278 - 280 (2013).

[5] Z. Li, R.J. Evans and B. Wittenmark: Minimum variance prediction of linear time-varying systems, Automatica, vol. 33, no.4, pp907-618, (1997). 